\title{
Nanopore Technology: A Simple, Inexpensive, Futuristic Technology for DNA Sequencing
}

\author{
P. D. Gupta ${ }^{1}$
}

Received: 10 January 2016/ Accepted: 6 July 2016/Published online: 22 July 2016

(C) Association of Clinical Biochemists of India 2016

\begin{abstract}
In health care, importance of DNA sequencing has been fully established. Sanger's Capillary Electrophoresis DNA sequencing methodology is time consuming, cumbersome, hence become more expensive. Lately, because of its versatility DNA sequencing became house hold name, and therefore, there is an urgent need of simple, fast, inexpensive, DNA sequencing technology. In the beginning of this century efforts were made, and Nanopore DNA sequencing technology was developed; still it is infancy, nevertheless, it is the futuristic technology.
\end{abstract}

\section{The Technique}

Most of the current DNA sequencing technologies requires working with short snippets of DNA, typically 50-100 nucleotides long. These must be processed by large sequencers in a laboratory. The cumbersome process can take days to weeks to complete.

Nanopore technology takes advantage of the small, tunnel-like structures found in bacterial membranes. In nature, such pores allow bacteria to control the flow of nutrients across their membranes. A pore surrounded by proteins or peptides in a solid-state membrane which allows a single-molecule pass through it, is termed as nanopore. With the development of Nanopore technology, now it became easy to sequence single strand DNA molecules by passing through the nanopores.

\footnotetext{
P. D. Gupta

pdg2000@hotmail.com

1 Manipal University, Manipal, Karnataka, India
}

The nanopores were created in an insulating membrane separating two chambers filled with conductive electrolyte. Charged molecules are driven through the pore under an applied electric potential (a process known as electrophoresis) by modulating the ionic current through the nanopore. This current reveals useful information about the structure and dynamic motion of the molecule.

When DNA strands are fed through a nanopore with a voltage difference across the pore, each molecule in the DNA strand by the amount of current that flows across the nanopore can be identified. The proper sequence of the bases adenine, thymine, guanine, and cytosine which connect single strands of DNA into a double helix can be ascertain.

The main problem with this method is that the distance between bases in the DNA strands are about half a nanometer. Therefore, if the nanopore is thicker than half a nanometer, individual bases in the DNA strand cannot be measured. For that reason, researchers must use material that is only one atom thick to make up the nanopore. Graphene sheets come to the rescue. These sheets are only one carbon atom thick, so nanopores made of graphene are thin enough to resolve individual bases in DNA strands. The probes are designed to bind with a sequence of DNA that is suspected of having a mutation. This can be done by creating a complimentary sequence of DNA to the doublehelix strand in question.

Then allow molecules containing both sequences to mix in a test tube in salt water, where they naturally will match up to one another if the base pairs are intact.

Unlike previous technologies, the probe molecule checks both strands of the target double helix for mutations rather than just one, which explains the increased specificity. 
The probe is engineered to emit a fluorescent glow if there's a perfect match between it and the target. If it doesn't illuminate, that means the strands didn't match and there was in fact a mutation in the target strand of DNA.

\section{Identification of Methylated DNA}

Two DNA bases, 5-methylcytosine (5mC) and 5-hydroxymethylcytosine $(5 \mathrm{hmC})$, marks epigenetic modification, are recognized in immobilized DNA strands and distinguished from G, A, T and C by nanopore current recording, the approach will provide epigenetic modifications in unamplified genomic DNA.

\section{Decoding Long DNA Strands}

Recently developed nanopore DNA sequencing technology could possibly be used to create 'tricorder'-like devices for detecting pathogens or diagnosing genetic disorders rapidly and on-the-spot, since it is capable of reading long sequences of DNA far more quickly than any existing techniques. Laszlo and Gundlach (Natue, 2014) used Mycobacterium smegmatis porin A (MspA). This bacterial pore has been genetically altered so that the narrowest part of the channel has a diameter of about a nanometer, or 1 billionth of a meter. This is large enough for a single strand of DNA to pass through. The modified nanopore is then inserted into a membrane separating two salt solutions to create a channel connecting the two solutions and a small voltage is applied across the membrane to make the ions of the salt solution flow through the nanopore. The ion flow creates a measurable current. If a strand of DNA is added to the solution on one side of the membrane and then enters a pore, the bulky DNA molecules will impede the flow of the much smaller ion and thereby alter the current. How much the current changes depend on which nucleotidesthe individual molecules adenine, guanine, cytosine and thymine that make up the DNA chain-are inside the pore. Detecting changes in current can reveal which nucleotides are passing through the nanopore's channel at any given instant. 